\title{
Study of correlations between CT properties of retrieved cerebral thrombi with treatment outcome of stroke patients
}

\author{
Rebeka Viltuznik ${ }^{1}$, Jernej Vidmar ${ }^{1,2}$, Andrej Fabjan²,3, Miran Jeromel ${ }^{4,5}$, Zoran V. \\ Milosevic ${ }^{4}$, Igor J. Kocijancic ${ }^{4}$, Igor Sersa ${ }^{1,2}$ \\ 1 Jožef Stefan Institute, Ljubljana, Slovenia \\ 2 Institute of Physiology, Medical Faculty, University of Ljubljana, Slovenia \\ ${ }^{3}$ Department of Vascular Neurology and Intensive Therapy, University Medical Centre Ljubljana, Slovenia \\ ${ }^{4}$ Department of Diagnostic and Interventional Neuroradiology, Clinical Institute of Radiology, University Medical Centre \\ Ljubljana, Slovenia \\ ${ }^{5}$ Department of Diagnostic and Interventional Radiology, General Hospital Slovenj Gradec, Slovenia
}

Radiol Oncol 2021; 55(4): 409-417.

Received 30 November 2020

Accepted 2 September 2021

Correspondence to: Igor Serša, Ph.D., Jožef Stefan Institute, Jamova 39, SI-1000 Ljubljana, Slovenia. E-mail: igor.sersa@ijs.si

Disclosure: No potential conflicts of interest were disclosed.

This is an open access article under the CC BY-NC-ND license (http://creativecommons.org/licenses/by-nc-nd/4.0/).

Background. All the patients with suspected stroke are directed to whole-brain CT scan. The purpose of this scan is to look for early features of ischemia and to rule out alternative diagnoses than stroke. In case of ischemic stroke, CT diagnostics (including CT angiography) is used mainly to locate the occlusion and its size, while the Hounsfield Units (HU) values of the thrombus causing the stroke are usually overlooked on CT scan or considered not important. The aim of this study was to demonstrate that the $\mathrm{HU}$ value is relevant and can help in better treatment planning.

Patients and methods. There were 25 patients included in the study, diagnosed with ischemic stroke in the middle cerebral artery (MCA) territory. In all patients, systemic thrombolysis was not successful and the mechanical recanalization was needed. The retrieved thrombi were also analyzed histologically for the determination of red blood cells (RBC) proportion. CT of the proximal MCA (M1) segment was analyzed for average HU value and its variability both in the occluded section and the symmetrical normal site. These CT parameters were then statistically studied for the possible correlations with different clinical, histological and procedure parameters using the Linear Regression and the Pearson correlation coefficient.

Results. Relevant positive correlations were found between average HU value of thrombus and outcome modified Rankin Scale (mRS), initial mRS, number of passes with thrombectomy device as well as RBC proportion.

Conclusions. Results of the present study suggest that measured HU values in CT images of the cerebral thrombi may help in the assessment of thrombus compaction and therefore better treatment planning.

Key words: ischemic stroke; CT images; cerebral thrombi; modified Rankin Scale; RBC proportion

\section{Introduction}

The risk for stroke is increasing yearly due to the aging population on a global level and the accumulation of risk factors. ${ }^{1}$ Stroke is the second leading cause of death and the main cause for decreased quality of life. ${ }^{2}$ Time is of the essence when it comes to treating the stroke, where thrombolytic treatment, usually with systemic administration of recombinant tissue plasmin activator (rt-PA), and mechanical thrombectomy are the main approaches; however new experimental treatments are also under consideration. ${ }^{3}$

The main cause of stroke is the acute cerebral artery occlusion, which leads to ischemic stroke, where the oxygen supply is cut off. ${ }^{4}$ The occlusion 
can either be thrombotic or embolic, originating from various locations, which results in various thrombi structures. The structure can also give us information on the age of the thrombi. ${ }^{5}$ On a microscopic level, thrombi are composed of different levels of red and white blood cells intertwined with fibrin meshwork. ${ }^{6,7}$ The structure can strongly influence the permeability which is important for the success of the thrombolytic treatment. ${ }^{8}$

The diagnosis of stroke is a multiple step process, which consists of clinical evaluation and diagnostic imaging. The clinical evaluation includes the assessment of the effect of the stroke, which can be estimated using the modified Rankin Scale (mRS). This is a seven-level stroke scale ranging from 0 , which stands for no symptoms to 6 , which stands for death. ${ }^{9}$ Another scale serving the same purpose, however, with more grades is National Institutes of Health (NIH) severity stroke scale (NIHss). ${ }^{10}$

In diagnostics of stroke, computed tomography (CT) is the main tool in selection of patients that are suitable candidates for stroke treatment: firstly, to exclude hemorrhage, to exclude large areas of hypodense brain tissue, suggesting irreversible ischemia and to exclude stroke mimics. Native CT is complemented by CT angiography (CTA), which provides high diagnostic value in the detection of occlusion in high degree of stenosis as well as CT perfusion (CTP), which provides high specificity in the detection of ischemia and infarcted brain tissue. However, the most accurate assessment for acute stroke involving the site of occlusion, infarction core and salvageable brain tissue is a combination of different CT procedures involving CTA and CTP. In addition, CT scanning is fast, it is widely accessible and its price per scan is relatively low. However, sensitivity of CT to soft tissues cannot match with that of MRI. ${ }^{11-13}$ While it has been proven that CT can be used to determine some characteristics of the thrombi ${ }^{14}$, the field of connecting CT characteristics to clinical and procedure parameters has yet to be investigated.

The aim of this study is to demonstrate that CT images provide more information than currently used in routine stroke diagnostics. More specifically, mainly geometrical and macroscopic parameters of CT images are used, while information on the $\mathrm{HU}$ values of the thrombus causing the ischemic stroke, are usually overlooked or considered not important. In this study we want to show that this information is relevant for assessment of the thrombus microscopic structure and that these parameters correlate with some clinical parameters and can be therefore used for better treatment planning.

\section{Patients and methods}

\section{Patient selection and stroke protocol}

This study was performed on $n=25$ patients which were diagnosed with acute ischemic stroke and underwent mechanical thrombectomy procedure with successful removal of the thrombus from the middle cerebral artery (MCA) M1 segment. Mean age of the patients was $73 \pm 11$ years and the group of patients consisted of 16 males and 9 females. In 11 patients, etiology of the stroke was atherothrombotic and in 14 patients it was cardioembolic. Before the stroke event four patients were receiving antiaggregating drugs, two patients were receiving anticoagulating drugs and one patient was receiving both types of drugs (Table 1).

The patients for the study were admitted to the Neurology Clinic of University Medical Center in Ljubljana for urgent neurological symptoms suggesting brain stroke. These patients were managed according to the standard steps of acute ischemic stroke management in our tertiary center. Firstly, an urgent clinical examination was performed, which was followed by a CT scan (non-contrast enhanced CT scan, CT perfusion and CT angiography) on a Siemens Sensation Open 40 CT scanner, where ischemic stroke caused by the occlusion of the middle cerebral artery, was confirmed. The protocol continued with standard full dose of rt-PA $(0.9 \mathrm{mg} / \mathrm{kg}$, maximum $90 \mathrm{mg})$ systemic thrombolytic treatment. In all studied patients clinical stroke signs persisted after the thrombolytic treatment, therefore further therapy was done by the mechanical thrombectomy. This was performed by skilled interventional neuroradiologist, using the standard mechanical recanalization procedure with the thrombectomy device (Trevo®stent retriever, $4 \mathrm{x}$ $20 \mathrm{~mm}$, Stryker Neurovascular, Kalamazoo, MI). The retrieved thrombi were preserved and additionally examined through histological analysis.

The protocol of the study was approved by the Institutional Review Board and the Ethical Committee of the National Ministry of Health of the Republic of Slovenia, approval No. 012099/2021/7. The study was performed in agreement with the informed-consent policy.

\section{CT imaging protocol and image analysis}

Stroke patients with qualifying conditions for the study underwent urgent CT scanning of the brain which included non-contrast enhad (NCE) sequential CT scans and CTA scans. The NCE CT scan is a sequential scan consisting of two parts-the skull 
TABLE 1. Experimental data of patients qualified for the study, which include CT, histological, clinical and procedure parameters

\begin{tabular}{|c|c|c|c|c|c|c|c|c|c|c|c|c|c|c|c|c|c|c|c|c|}
\hline \multirow{3}{*}{$\#$} & \multicolumn{9}{|c|}{ СT parameters } & \multirow{2}{*}{ Histology } & \multicolumn{8}{|c|}{ Clinical parameters } & \multirow{2}{*}{\multicolumn{2}{|c|}{$\begin{array}{l}\text { Procedure } \\
\text { parameters }\end{array}$}} \\
\hline & \multicolumn{4}{|c|}{ HU avg } & \multicolumn{4}{|c|}{ HU var } & \multirow[b]{2}{*}{$\begin{array}{c}\mathrm{L} \\
{[\mathrm{mm}]}\end{array}$} & & \multirow{2}{*}{$\begin{array}{l}\text { - Stroke } \\
\text { etiology }\end{array}$} & \multirow{2}{*}{$\begin{array}{l}\text { Therapy } \\
\text { before } \\
\text { stoke }\end{array}$} & \multicolumn{3}{|c|}{ NIHSS } & \multicolumn{3}{|c|}{ mRS } & & \\
\hline & occl & nor & diff & $\begin{array}{l}\text { diff } \\
\text { [\%] }\end{array}$ & occl & nor & diff & $\begin{array}{l}\text { diff } \\
\text { [\%] }\end{array}$ & & $\begin{array}{l}\text { RBC } \\
{[\%]}\end{array}$ & & & strt & end & diff & strt & end & diff & $\begin{array}{c}\mathrm{Rd} \\
\text { [min] }\end{array}$ & \# pass \\
\hline 1. & 29.1 & 32.4 & -3.3 & -11.4 & 4.20 & 4.42 & -0.22 & -5.0 & 20.8 & 11.5 & AT & 1 & 21 & 1 & 20 & 4 & 1 & 3 & 42 & 1 \\
\hline 2. & 42.0 & 29.2 & 12.8 & 30.5 & 4.58 & 8.98 & -4.40 & -96.3 & 16.3 & 45.1 & CE & 1 & 17 & 8 & 9 & 5 & 4 & 1 & 62 & 2 \\
\hline 3. & 38.5 & 35.1 & 3.4 & 8.9 & 3.56 & 2.75 & 0.80 & 22.6 & 17.0 & 12.0 & CE & I & 23 & 7 & 16 & 4 & 3 & 1 & 65 & 1 \\
\hline 5. & 43.7 & 44.2 & -0.5 & -1.2 & 3.55 & 3.86 & -0.31 & -8.7 & 17.9 & 65.9 & AT & I & 26 & 6 & 20 & 5 & 3 & 2 & 97 & 3 \\
\hline 6. & 35.8 & 30.0 & 5.9 & 16.4 & 4.17 & 2.23 & 1.95 & 46.7 & 16.3 & 61.0 & CE & 1 & 7 & 0 & 7 & 3 & 0 & 3 & 38 & 3 \\
\hline 7. & 31.8 & 36.0 & -4.2 & -13.2 & 2.77 & 3.26 & -0.49 & -17.8 & 22.2 & 57.3 & AT & 1 & 13 & 3 & 10 & 4 & 1 & 3 & 90 & 1 \\
\hline 8. & 37.8 & 38.9 & -1.1 & -2.9 & 2.82 & 4.45 & -1.63 & -58.0 & 26.9 & 50.3 & CE & 1 & 14 & 3 & 11 & 4 & 3 & 1 & 77 & 1 \\
\hline 12. & 35.7 & 33.9 & 1.7 & 4.9 & 3.56 & 5.39 & -1.83 & -51.2 & 13.9 & 31.8 & CE & AA & 6 & 3 & 3 & 3 & 1 & 2 & 69 & 1 \\
\hline 13. & 44.3 & 31.9 & 12.3 & 27.8 & 4.66 & 5.11 & -0.46 & -9.8 & 22.5 & 74.7 & AT & 1 & 19 & 12 & 7 & 5 & 4 & 1 & 108 & 2 \\
\hline 14. & 44.1 & 37.5 & 6.6 & 15.0 & 2.60 & 3.92 & -1.32 & -50.9 & 19.3 & 38.9 & CE & I & 18 & 20 & -2 & 4 & 5 & -1 & 63 & 5 \\
\hline 15. & 36.0 & 35.4 & 0.6 & 1.6 & 3.48 & 2.67 & 0.81 & 23.4 & 17.9 & 48.8 & CE & 1 & 42 & 42 & 0 & 5 & 6 & -1 & 75 & 2 \\
\hline 16. & 38.1 & 36.4 & 1.7 & 4.5 & 4.16 & 5.45 & -1.30 & -31.2 & 18.6 & 21.3 & AT & 1 & 15 & 4 & 11 & 5 & 4 & 1 & 77 & 1 \\
\hline 17. & 42.5 & 40.7 & 1.8 & 4.2 & 3.11 & 3.74 & -0.63 & -20.4 & 13.5 & 14.8 & $\mathrm{CE}$ & $\mathrm{AA}$ & 14 & 2 & 12 & 5 & 3 & 2 & 53 & 1 \\
\hline 18. & 38.7 & 23.3 & 15.4 & 39.8 & 5.66 & 4.79 & 0.87 & 15.3 & 19.1 & 42.4 & CE & $A C$ & 22 & 3 & 19 & 5 & 4 & 1 & 43 & 1 \\
\hline 19. & 34.7 & 30.9 & 3.8 & 11.0 & 5.61 & 4.43 & 1.18 & 21.1 & 20.9 & 37.7 & AT & 1 & 3 & 1 & 2 & 1 & 0 & 1 & 60 & 1 \\
\hline 20. & 41.2 & 32.4 & 8.8 & 21.4 & 5.14 & 4.58 & 0.56 & 10.9 & 19.3 & 56.0 & AT & $A C$ & 11 & 40 & -29 & 4 & 6 & -2 & 76 & 3 \\
\hline
\end{tabular}

$\mathrm{AA}=$ antiaggregation; $\mathrm{AC}=$ anticoagulant; $\mathrm{ACAA}=$ both types of drugs; $\mathrm{AT}$ = atherothrombotic; $\mathrm{CE}=$ cardioembolic; diff = absolute difference; diff $[\%]=$ relative difference in \% HU avg = average Hounsfield units; $\mathrm{HU}$ var = variability of Hounsfield units; L $[\mathrm{mm}]=\mathrm{CT}$ length of the thrombi; $\mathrm{mRS}=$ modified Rankin score; NIHSS = NIH Stroke Scale; nor = normal artery; occl = occlusion; RBC [\%] = percentage of red blood cells in the thrombi; $\mathrm{Rd}=$ duration of mechanical recanalization; \# pass = number of passes

base $(120 \mathrm{kV}, 265 \mathrm{mAs}$, matrix $1024 \times 1024$, slice thickness $3 \mathrm{~mm}$, collimation $20 \times 0.6$, rotation time 1 s, window width 90-190, window center 38) and the cerebral part $(120 \mathrm{kV}, 310 \mathrm{mAs}$, matrix $1024 \times 1024$, slice thickness $4.8 \mathrm{~mm}$, collimation $24 \times 1.2$, rotation time 1 s, window width 80 , window center 38 ). Acquired CT images were further analyzed by the ImageJ program (NIH, Bethesda MD, USA) to obtain relevant $\mathrm{CT}$ data on brain thrombi of the patients. CTA images were specifically used to determine the position of the thrombi. This information was then used to stack three slices from NCE CT scan containing the thrombus to correctly position the line along the thrombus on the stacked image as well as the symmetrical non-occluded MCA segment on the opposite side of the brain. Special care was taken to center the lines in the middle of the vessel in order to avoid signals from the vessel wall tissue and therefore reduce a possible partial volume effect and also an increased Hounsfield Units (HU) values due to vessel calcifications. Along the lines the HU intensity profiles were measured in the NCE CT images. NCE CT images were more suitable for the thrombus analysis than CTA images, because they have no contrast enhancement due to the contrast agent that could alter the HU of thrombi. Measured HU intensity profiles were further analyzed by determining average $\mathrm{HU}$ val- 
ue of the profile $H U_{-} a v g$ and its standard deviation HU_var. HU_avg and HU_var values of thrombi occluded (ocl) were compared with the corresponding counterpart values of the non-occluded (nor) symmetric MCA segment.

\section{Interventional procedure parameters and clinical parameters}

During every interventional procedure, recanalization time and number of passes with the thrombectomy device were registered as the procedure parameters. The recanalization time was considered as the time between the first contact of the thrombectomy device with the thrombus to the successful recanalization through the occluded artery with complete removal of the thrombus.

For clinical parameters, modified Ranking Scale $(m R S)$ for stroke before ( $m R S$ _start, assessed at admission to the hospital) and after the procedure (mRS_end, assessed at discharge from the hospital) was collected and the $m R S$ difference between these two parameters $\left(m R S \_d i f f=m R S \_e n d-m R S\right.$ _ start) was calculated.

\section{Histological analysis}

Histological analysis was done to determine the percentage of red blood cells (RBC) in the retrieved thrombi (RBC\%). The thrombi samples were fixed in $10 \%$ buffered formaldehyde for $48 \mathrm{~h}$. After the fixation, they were cut longitudinally as $5-\mu \mathrm{m}$ thick cross-sections and embedded in paraffin. The cross-sections were stained with monoclonal antibodies Anti-Human Glycophorin A (GPA) for RBC content and with anti $\beta-3$ integrin Anti-Human CD61 (DakoCytomation, Denmark) for platelet content.

Micro-photography of the stained cross-sections was performed, using a Nikon Eclipse E600 optical microscope (Nikon, Düsseldorf, Germany) equipped with a Nikon 4x Plan Fluor objective and with a high-resolution CCD camera Nikon DS-Fi1. The micro-photography system was controlled by the Nikon NIS Elements software package. The exposure time yielding the optimal image contrast was equal to $10 \mathrm{~ms}$ while the in-plane image resolution was equal to $10 \mu \mathrm{m}$ (imaging matrix was $1024 \times 1124$ and field of view (FOV) $10.24 \times 11.24$ $\mathrm{mm}^{2}$ ).

Histological (hematoxylin-eosin) images of the central cross-section along the thrombi were examined for the RBC proportion by the analysis encompassing the following steps. First, each image was corrected for uneven illumination (vignetting). ${ }^{15}$ Then the corresponding intensity histogram was calculated and used to determine the optimal threshold for the discrimination between the RBCrich and the platelet-rich regions. The RBC proportion was determined as the ratio between the thresholded RBC area and the total thrombi area.

\section{Statistical analysis}

Possible correlations between different groups of data (CT image parameters, histological parameters, clinical parameters and procedure parameters) were tested. Univariate linear regression was the statistical method of choice, where $R^{2}$, linear regression coefficient with its standard error and $p$-value were calculated for all the possible pairs of data groups. In addition to the regression analysis, Pearson correlation coefficient was calculated for all the tested data pairs. Statistical analysis was performed using Microsoft Excel Analysis ToolPak software.

\section{Results}

Histological sections of selected representative retrieved cerebral thrombi are shown in Figure 1. Histological slices were stained by hematoxylineosin and then analyzed for RBC proportions using digital image processing of the thrombi images acquired by using optical microscopy of the histological sections. All retrieved thrombi had a distinctly heterogeneous, laminated (multilayer) structure. The structure involves the interweaving of compacted erythrocyte-rich (red) regions with thinner (pink) coatings containing a combination of complementary-linked platelets and a fibrin network. The laminations are often folded and twisted, which is probably due to blood flow turbulence in the environment where the thrombi were formed and their turning as they traveled along the vessel. Among the retrieved thrombi there were no homogeneous single-layered (red only) thrombi. Which can be explain by their high susceptibility to thrombolysis.

Figure 2 shows an example of a CT image of a stroke patient with a clearly visible MCA segment on both hemispheres of the brain. In the example the MCA segment on the right hemisphere is occluded by the cerebral thrombus, while the MCA segment on the opposite hemisphere is normal. Exact location of each thrombus was determined from its CTA image first and then this location was 

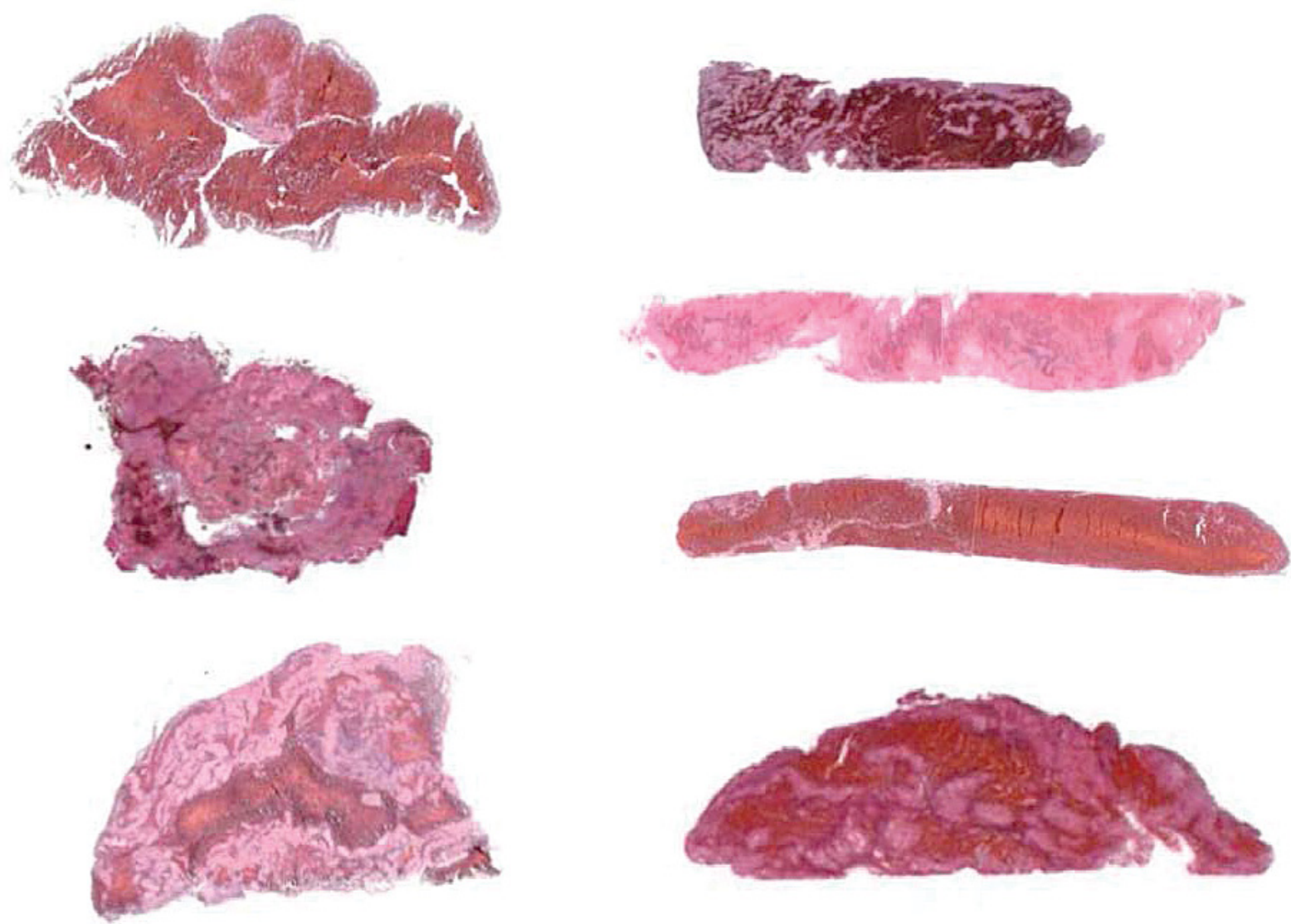

FIGURE 1. Light microscopy images of histological slices along representative retrieved cerebral thrombi. The histological slices were stained by hematoxylin-eosin and then analyzed for the RBS proportion. It can be seen from the images that the structural and compositional diversity among cerebral thrombi is high. All retrieved thrombi had a distinctly heterogeneous, laminated (multilayer) structure which involves the interweaving of compacted erythrocyte-rich (red) regions with thinner (pink) coatings containing a combination of complementary-linked platelets and a fibrin network.

used to obtain HU values along the thrombus from the corresponding CT image. Yellow line on the image is drawn along the thrombus and the symmetrical position of the normal MCA segment. Graphs in Figure 2A correspond to HU intensity profiles along both yellow lines: along the cerebral thrombus in Figure 2B and along the symmetrical section of the normal MCA segment in Figure 2C. For each $\mathrm{HU}$, intensity profile was then calculated by average $\mathrm{HU}$ value $H U_{-} a v g$ and $\mathrm{HU}$ value variability $H U \_v a r$. Thus, each patient was characterized by these two CT parameters that were obtained for the occluded (occl) and normal (nor) MCA segment so that it became possible to calculate their absolute (diff) and relative differences (diff [\%]), which are equal to: HU_avg_diff = HU_avg_occl-HU_avg_nor, $H U \_a v g \_d i f f[\%]=100 \cdot\left(1-H U \_a v g \_n o r / H U \_a v g \_o c c l\right)$ and HU_var_diff = HU_var_occl-HU_var_nor, $H U_{-} v a r \_d i f f[\%]=100 \cdot\left(1-H U \_v a r \_n o r / H U \_v a r \_o c c l\right)$. Table 1 shows CT as well as histological (RBC proportion), clinical and procedure parameters of pa-
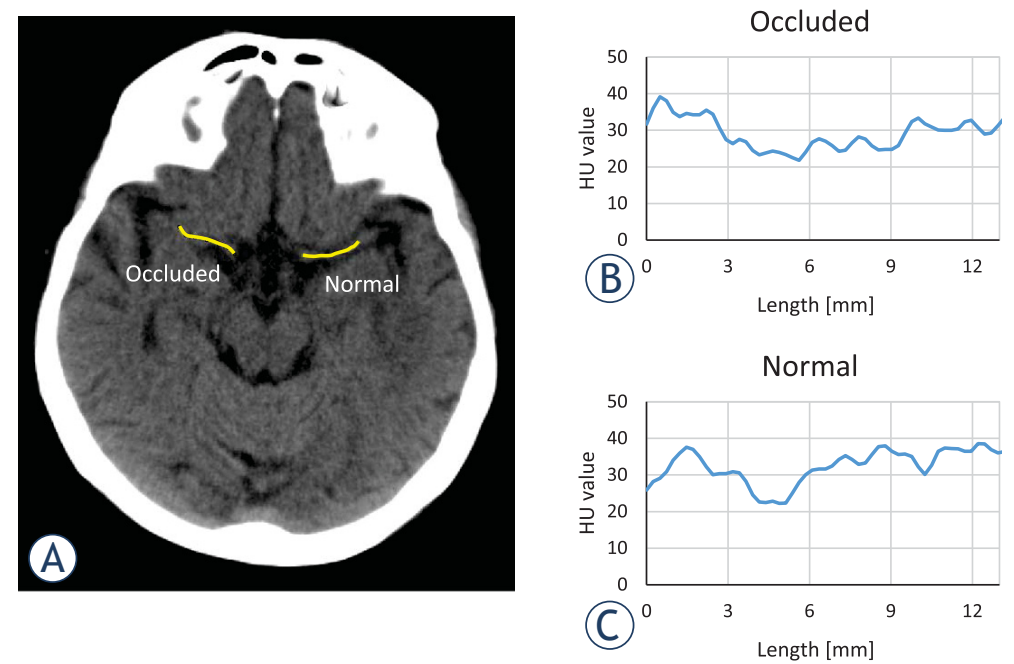

FIGURE 2. CT image of a patient with stroke (A). In the selected slice, Middle Cerebral Artery (MCA) on both hemispheres of the brain is visible. The occluded MCA section on the right hemisphere and its symmetrical normal MCA section is indicated by a yellow line along the cerebral thrombus. Graphs show Hounsfield Units (HU) value profiles of the CT image along the lines for the occluded (B) and the normal (C) MCA segment. 
TABLE 2. Linear regression and Pearson correlation coefficient analysis of data group pairs with statistically most significant correlations and linear regression parameters

\begin{tabular}{cccccc}
\hline \multicolumn{2}{c}{ Data group pair } & \multicolumn{3}{c}{$\begin{array}{c}\text { Linear regression } \\
\boldsymbol{y}=\boldsymbol{k} \cdot \boldsymbol{x}+\boldsymbol{n}\end{array}$} & $\begin{array}{c}\text { Pearson } \\
\text { coefficient }\end{array}$ \\
\hline $\mathrm{x}$-values & $\boldsymbol{y}$-values & $\boldsymbol{k}$ & $\boldsymbol{p}$-value & $\boldsymbol{R}^{2}$ & $\boldsymbol{\rho}$ \\
\hline $\mathrm{HU}$ avg occl & mRS end & $0.227 \pm 0.086$ & 0.015 & 0.233 & 0.483 \\
$\mathrm{HU}$ avg occl & \# passes & $0.119 \pm 0.052$ & 0.031 & 0.186 & 0.432 \\
$\mathrm{HU}$ avg occl & mRS diff & $-0.140 \pm 0.067$ & 0.049 & 0.158 & -0.398 \\
$\mathrm{HU}$ avg diff & RBC [\%] & $1.646 \pm 0.809$ & 0.053 & 0.153 & 0.391 \\
$\mathrm{HU}$ avg occl & mRS start & $0.087 \pm 0.045$ & 0.065 & 0.140 & 0.374 \\
$\mathrm{HU}$ avg diff & mRS diff & $-0.104 \pm 0.059$ & 0.093 & 0.118 & -0.343 \\
\hline
\end{tabular}

$\mathrm{HU}$ avg = average Hounsfield Units; occl = occluded MCA segment; diff = absolute difference: $\mathrm{mRS}=$ modified Rankin score; $\mathrm{RBC}[\%]=$ percentage of red blood cells in the thrombi

tients and their retrieved thrombi included in the study.

Correlation between different pairs of data groups from Table 1 were tested by the Pearson correlation coefficient and the univariate linear regression analysis. Results of this analysis for the pairs with the highest correlation is shown by linear regression graphs in Figure 3 and their corresponding correlation and linear regression parameters are formulated in Table 2 . The linear
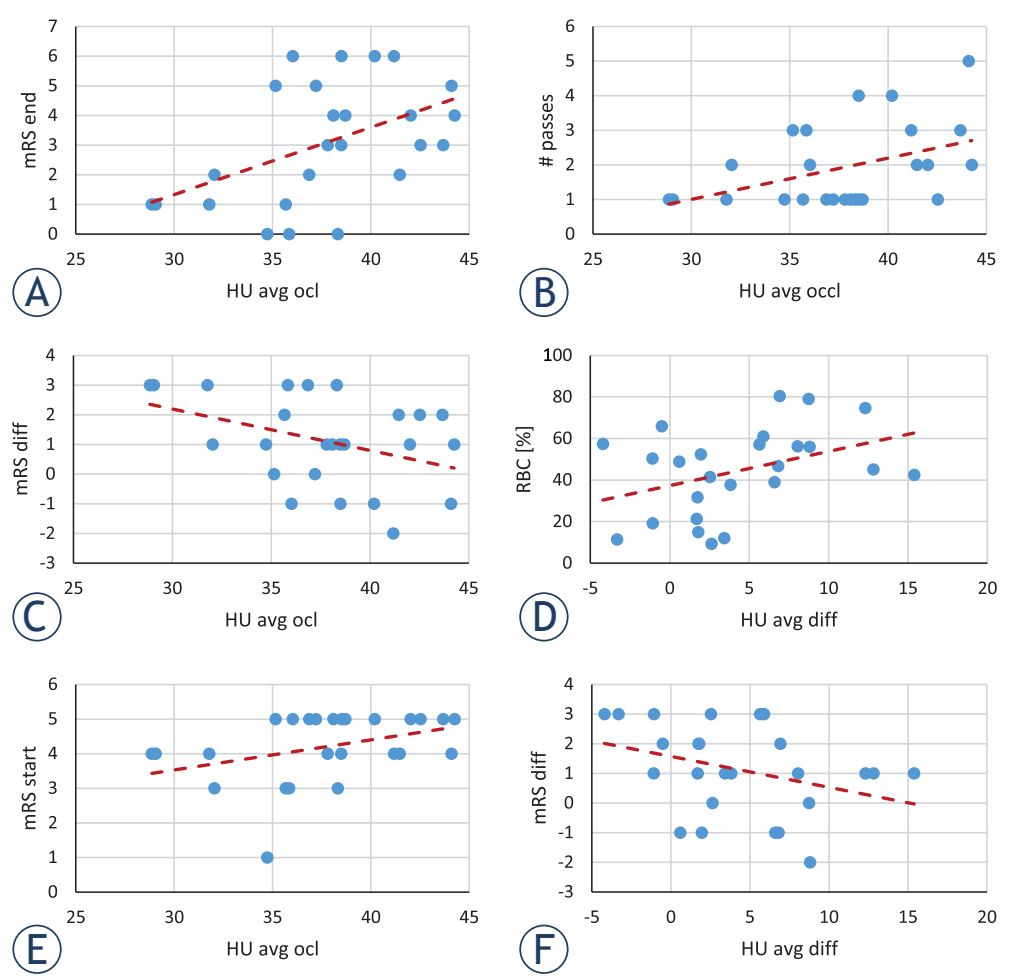

FIGURE 3. Graphs of correlation among different data groups. Shown are six graphs of data group pairs from Table 2 that have the highest Pearson coefficients and also statistically most significant linear regression parameters. regression statistical analysis showed significant differences $(p<0.05)$ only in three cases, i.e., for the group pars: outcome mRS vs. average HU in the occluded MCA, the number of passes with the thrombectomy device vs. the average $\mathrm{HU}$ in the occluded MCA, and the difference between the initial and the outcome mRS vs. the average HU in the occluded MCA. The next three pairs, also included in Table 2 and Figure 3, had $0.05<p<0.1$ which can be considered as borderline significant. These pairs are: thrombus RBC proportion vs. the difference between the average HU in the occluded and normal MCA, initial mRS vs. the average HU in the occluded MCA and the difference between the initial and the outcome mRS vs. difference between average $\mathrm{HU}$ in the occluded and normal MCA. From the graphs in Figure 3A,E it can be inferred that the average HU in the occluded MCA is in positive correlation with both the initial and the outcome mRS. As the regression coefficient (slope $k$ ) is higher for the outcome mRS than the initial mRS, the correlation between difference of the initial and the outcome $\mathrm{mRS}$ ( $m R$ S_diff $=m R S \_s t a r t-m R S \_$_nd $)$ and average $H U$ in the occluded MCA is negative (Figure 3C). The number of passes of the thrombectomy device is also in positive correlation with the average HU in the occluded MCA (Figure 3b). The remaining two of the more significant correlations involve differentiation between the average HU in the occluded and the normal MCA which is in positive correlation with the RBC proportion (Figure 3D); and the negative correlation with the difference of mRSs (Figure 3F). From the results in Table 2, it can also be seen that the data group pairs with higher coefficients of determination $R^{2}$ in the linear regression analysis also have higher Pearson coefficients of correlation. Therefore, $R^{2}$ can also be considered a measure to the extent of correlation.

\section{Discussion}

CT was already employed to study properties of artificially made blood clots. In a study by Kirchhof et al. it was concluded that CT has a potential to differentiate between clots with high fibrinogen content and those with low content, but is unable to distinguish between pure plasma clots and low hematocrit clots. ${ }^{16}$ In a more recent study by Brinjikji et al. single-energy and dual-energy CT were compared in their power to differentiate among different types of artificial blood clots. ${ }^{17}$ They confirmed superiority of dual-energy CT in differentiation between high and low RBC content blood clots. 
However, studies where CT properties of blood clots in vivo are studied are relatively scarce. ${ }^{18}$ In our study on correlations between CT properties of MCA thrombi and thrombectomy procedure parameters, treatment outcome, and histology the highest correlation was found between average HU in the occluded MCA (HU_avg_occl) and the stroke outcome assessed with mRS (mRS_end). According to our findings, higher average $\mathrm{HU}$ in the occluded MCA suggests higher mRS. This is because the most important factor among the factors that contribute to the assessment of mRS (such as extent of penumbra tissue, collateral vasculature, time form onset of the occlusion to the intervention, etc.) is the morphology of the thrombus, especially its compaction. Due to their retraction, thrombi with higher compaction, which are often older with low serum content ${ }^{19,20}$ have higher average HU values, while fresh thrombi with more serum spaces ${ }^{21}$, have lower overall HU.

The correlation between the number of passes with the thrombectomy device and average $\mathrm{HU}$ of the thrombi can also be associated with the thrombi organization. More organized (hard, fibrin-rich) thrombi can attach strongly with the arterial wall, which may result in the more difficult retrieval of such thrombus during the interventional procedure. ${ }^{22}$ This suggest, that if higher average HU values in the MCA occlusion are found on the CT scan, it is getting more likely that more than one pass will be needed to successfully retrieve the thrombus during the interventional procedure.

When treating patients with stroke, time is essential. ${ }^{23}$ The longer time taken till reestablishment of the normal cerebral blood flow, the less oxygen is present in the brain, which may result in brain cell and nerve connection loss. This contributes to more serious symptoms and a higher mRS classification over time. ${ }^{23}$ Outcome after mechanical thrombectomy can be more dependent on the capacity of collateral circulation than on the time from stroke onset. ${ }^{24}$ However, time still plays an important role in saving tissue at risk (penumbra). In addition, the longer time taken till resolving the occlusion, the thrombus can retract further and therefore becomes more compact with a low serum content. Clot retraction is an important contributing factor for the correlation that was found between the initial mRS (mRS_start before the treatment) and the average HU of the thrombus (HU_avg_occl). This factor may also explain the correlation between the average $\mathrm{HU}$ of the thrombus (HU_avg_occl) and the mRS classification difference between the initial and the outcome scores (mRS_diff). As already pointed out, a poorer treatment outcome can be expected in patients with poor collateral status. ${ }^{25}$ However, time since the formation of thrombus also impacts its properties, especially its retraction, which increases with the time since thrombus formation. ${ }^{26}$ The correlation between the average $\mathrm{HU}$ in the occluded MCA and the difference between the initial and the outcome mRS is negative (Figure 3C). This is because patients with a high average HU in the occluded MCA have usually higher initial mRS classification and their outcome mRS, on average is only slightly lower or even equal to the initial mRS due to the poor treatment results; e.g. patients with the initial $\mathrm{mRS}$ of 5 have often the outcome mRS of 4 or 5 so that the mRS difference ( $m R S$ _diff) is 0 or 1 , which corresponds to a slim or no treatment improvement. In patients with low average HU in occluded MCA, the initial $\mathrm{mRS}$ classification is lower and therefore, their outcome $\mathrm{mRS}$ is even much lower, e.g., patients with the initial mRS of $1-3$ can recover to mRS of 0 or 1 , so that the mRS difference is in the range $0-3$. The treatment outcome in these patients is better due to the short time frame from thrombus formation to intervention which is associated with earlier reperfusion and smaller brain tissue damage. ${ }^{27}$

In addition to average $\mathrm{HU}$ in the occluded MCA segment (HU_avg_occl), its difference to the corresponding value in normal MCA segment (HU_avg_ diff) was measured as well. The average HU difference value has in principle advantage over the single-sided MCA values (HU_avg_occl and $\mathrm{HU}_{-}$ avg_nor) as it is insensitive to possible offsets in HU values in CT images. The offsets cancel out in the subtraction of average $\mathrm{HU}$ values between the occluded and the normal MCA segments. Possible $\mathrm{HU}$ value offset can have an origin in image processing where consecutive three NCE CT image slices in the section with both MCA segments were stacked together. Therefore, the measured HU can in principle contain unwanted contributions from arterial wall and other tissues surrounding the vessel. In addition, the reference HU, that is measured in non-occluded symmetrical MCA segment, can be elevated due to possible arterial wall calcification, hematocrit level.

Positive correlation between RBC proportion $(R B C[\%])$ and the difference between average $\mathrm{HU}$ in the occluded and normal MCA (HU_avg_diff) can be explained by the presence of iron in RBCs. ${ }^{28}$ The thrombi with a higher proportion of RBC have a higher iron content and therefore absorb more $\mathrm{X}$-rays so that an increased HU values are detected in the CT images of these thrombi. In addition, 
thrombi with a higher proportion of RBC are also more compact, which further contributes to greater X-ray absorption. Another reason for increased HU values in the MCA region are also calcifications in the vessel wall, which cause higher X-ray absorption. If that is the case MCA may appear hyperdense also without thrombus in it.

Pretreatment thrombus evaluation can be of great clinical importance. Failure of thrombolytic treatment can be expected in fibrin rich thrombi. Thrombus composition can also have an impact on interventional treatment planning (mechanical thrombectomy). Optimal technique (stent retriever vs. aspiration) or device type selection can potentially be chosen on the basis of preoperative imaging data.

Major drawback of this study is limited number of cases $(n=25)$ and the use of sequential NCE CT. CT image resolution was insufficient to avoid the partial-volume effect in measurement HU profiles along the thrombi so that the HU profiles can be contaminated with HU values of the surrounding tissues. Specifically, in this study CT image analysis included stacking of three consecutive CT slices could result in inclusion of other brain tissues than the thrombus or normal MCA vessel because of the inferior bend of the MCA that can be anatomically present. Additional problem with the HU values of the vessel wall is that they can vary also due to possible atherosclerotic soft calcifications and these calcifications are often asymmetric, so that using the $\mathrm{HU}$ profile of the symmetric non-occluded MCA segment as the reference becomes questionable. If the spiral CT would be used instead of sequential CT, the CT images could also be processed in other (rotated) planes and the stacked slices could then be positioned and oriented ideally parallel to both MCA segments without compromising the quality of the observed vessel. This would make the HU measurements as well as the results more precise.

\section{Conclusions}

Routinely acquired CT images of stroke patients provide also information on HU values of thrombi that is usually ignored. In this study a relation between the HU value of a thrombus and its composition was confirmed. As more compact thrombus may represent a bigger problem for the interventional procedure a priori assessment of thrombus compaction is very important for good intervention planning. The present study also provides foundations for further studies where the accuracy of study could be improved by increasing the number of samples (patients and thrombi) and having more accurate spiral CT images of patients with stroke that would allow improve HU value determination in the targeted region.

\section{Acknowledgment}

The authors thank Kanza Awais for proofreading the manuscript. This study was financially supported by the Slovenian Research Agency grant J3-9288.

\section{References}

1. Virani SS, Alonso A, Benjamin EJ, Bittencourt MS, Callaway CW, Carson AP, et al. Heart disease and stroke statistics-2020 update: a report from the american heart association. Circulation 2020; 141: e139-596. doi: 10.1161/ CIR.0000000000000757

2. Tran J, Mirzaei M, Anderson L, Leeder SR. The epidemiology of stroke in the Middle East and North Africa. J Neurol Sci 2010; 295: 38-40. doi: 10.1016/j. jns.2010.05.016

3. Yamashita T, Abe K. Recent progress in therapeutic strategies for ischemic stroke. Cell Transplant 2016; 25: 893-8. doi: 10.3727/096368916X690548

4. Mozaffarian D, Benjamin EJ, Go AS, Arnett DK, Blaha MJ, Cushman M, et al. Heart disease and stroke statistics-2016 update: a report from the American Heart Association. Circulation 2016; 133: e38-360. doi: 10.1161/ CIR.0000000000000350

5. Chernysh IN, Nagaswami C, Kosolapova S, Peshkova AD, Cuker A, Cines $\mathrm{DB}$, et al. The distinctive structure and composition of arterial and venous thrombi and pulmonary emboli. Sci Rep 2020; 10: 5112. doi: 10.1038/ s41598-020-59526-X

6. Bajd F, Vidmar J, Fabjan A, Blinc A, Kralj E, Bizjak N, et al. Impact of altered venous hemodynamic conditions on the formation of platelet layers in thromboemboli. Thromb Res 2012; 129: 158-63. doi: 10.1016/j.thromres.2011.09.007

7. Bajd F, Vidmar J, Blinc A, Sersa I. Microscopic clot fragment evidence of biochemo-mechanical degradation effects in thrombolysis. Thromb Res 2010; 126: 137-43. doi: 10.1016/j.thromres.2010.04.012

8. Fang J, Tsui PH. Evaluation of thrombolysis by using ultrasonic imaging: an in vitro study. Sci Rep 2015; 5: 11669. doi: 10.1038/srep11669

9. Broderick JP, Adeoye O, Elm J. Evolution of the modified rankin scale and its use in future stroke trials. Stroke 2017; 48: 2007-12. doi: 10.1161/ STROKEAHA.117.017866

10. Kwah LK, Diong J. National Institutes of Health Stroke Scale (NIHSS). $J$ Physiother 2014; 60: 61. doi: 10.1016/j.jphys.2013.12.012

11. Kim BJ, Kang HG, Kim HJ, Ahn SH, Kim NY, Warach S, et al. Magnetic resonance imaging in acute ischemic stroke treatment. J Stroke 2014; 16: 13145. doi: 10.5853/jos.2014.16.3.131

12. Provost C, Soudant M, Legrand L, Ben Hassen W, Xie Y, Soize S, et al. Magnetic resonance imaging or computed tomography before treatment in acute ischemic stroke. Stroke 2019; 50: 659-64. doi: 10.1161/ STROKEAHA.118.023882

13. Dehkharghani S, Andre J. Imaging approaches to stroke and neurovascular disease. Neurosurgery 2017; 80: 991. doi: 10.1093/neuros/nyx268

14. Boodt N, Compagne KCJ, Dutra BG, Samuels N, Tolhuisen ML, Alves H, et al. Stroke etiology and thrombus computed tomography characteristics in patients with acute ischemic stroke: A MR CLEAN Registry Substudy. Stroke 2020; 51: 1727-35. doi: 10.1161/STROKEAHA.119.027749 
15. Leong FJ, Brady M, McGee JO. Correction of uneven illumination (vignetting) in digital microscopy images. J Clin Pathol 2003; 56: 619-21. doi: 10.1136/ jcp.56.8.619

16. Kirchhof K, Welzel T, Mecke C, Zoubaa S, Sartor K. Differentiation of white, mixed, and red thrombi: value of CT in estimation of the prognosis of thrombolysis phantom study. Radiology 2003; 228: 126-30. doi: 10.1148/ radiol.2273020530

17. Brinjikji W, Michalak G, Kadirvel R, Dai D, Gilvarry M, Duffy S, et al. Utility of single-energy and dual-energy computed tomography in clot characterization: an in-vitro study. Interv Neuroradiol 2017; 23: 279-84. doi: $10.1177 / 1591019917694479$

18. Liebeskind DS, Sanossian N, Yong WH, Starkman S, Tsang MP, Moya AL, et al. $\mathrm{CT}$ and MRI early vessel signs reflect clot composition in acute stroke. Stroke 2011; 42: 1237-43. doi: 10.1161/STROKEAHA.110.605576

19. Kozak M, Mikac U, Blinc A, Sersa I. Lysability of arterial thrombi assessed by magnetic resonance imaging. Vasa 2005; 34: 262-5. doi: 10.1024/03011526.34.4.262

20. Czaplicki C, Albadawi H, Partovi S, Gandhi RT, Quencer K, Deipolyi AR, et al. Can thrombus age guide thrombolytic therapy? Cardiovasc Diagn Ther 2017; 7: S186-96. doi: 10.21037/cdt.2017.11.05

21. Sabovic M, Lijnen HR, Keber D, Collen D. Effect of retraction on the lysis of human clots with fibrin specific and non-fibrin specific plasminogen activators. Thromb Haemost 1989; 62: 1083-7. doi: 10.1055/s-0038-1647122

22. Kim BM. Causes and solutions of endovascular treatment failure. J Stroke 2017; 19: 131-42. doi: 10.5853/jos.2017.00283

23. Chugh C. Acute ischemic stroke: management approach. Indian J Crit Care Med 2019; 23: S140-6. doi: 10.5005/jp-journals-10071-23192

24. Jeromel M, Milosevic ZV, Oblak JP. Mechanical recanalization for acute bilateral cerebral artery occlusion -literature overview with a case. Radiol Oncol 2020; 54: 144-8. doi: 10.2478/raon-2020-0017

25. Bang OY, Saver JL, Kim SJ, Kim GM, Chung CS, Ovbiagele B, et al. Collateral flow predicts response to endovascular therapy for acute ischemic stroke. Stroke 2011; 42: 693-9. doi: 10.1161/STROKEAHA.110.595256

26. ohnson S, Chueh J, Gounis MJ, McCarthy R, McGarry JP, McHugh PE, et al. Mechanical behavior of in vitro blood clots and the implications for acute ischemic stroke treatment. J Neurointerv Surg 2020; 12: 853-7. doi: 10.1136/ neurintsurg-2019-015489

27. Hacke W, Donnan G, Fieschi C, Kaste M, von Kummer R, Broderick JP, et al. Association of outcome with early stroke treatment: pooled analysis of ATLANTIS, ECASS, and NINDS rt-PA stroke trials. Lancet 2004; 363: 768-74. doi: 10.1016/S0140-6736(04)15692-4

28. Velasco Gonzalez A, Buerke B, Gorlich D, Fobker M, Rusche T, Sauerland C, et al. Clot analog attenuation in non-contrast CT predicts histology: an experimental study using machine learning. Trans/ Stroke Res 2020; 11: 940-9. doi: 10.1007/s12975-019-00766-z 